\title{
Synovial interlukin-6 affects apoptosis induction via nuclear factor kappa-B and fractalkine pathway during adjuvant arthritis
}

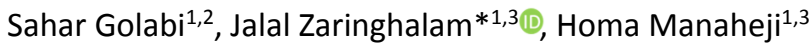 \\ Received: 23 Oct 2018 \\ Published: 25 Mar 2020
}

\section{Abstract}

Background: Apoptosis disruptions play substantial roles in pathogenesis of arthritis and its symptoms. Cytokines and their intracellular signaling have pivotal roles in arthritis pathophysiology. This study aimed to investigate the relationship between synovial Interleukin-6 (IL-6), nuclear factor kappa-B (NF-kB), and fractalkine (FKN) in the changes of edema and apoptosis during adjuvantinduced knee arthritis.

Methods: A total of 240 male Wistar rats were divided into different groups. Arthritis was evoked and the knee edema changes were evaluated by Vernier caliper. Synovial IL-6 was assayed by rat standard ELISA kit. Levels of NF-KB, fractalkine, and apoptotic indicators in the synovium were evaluated by Western blot method. Results were expressed as Mean \pm SEM. To analyze within-group variations, repeated measures ANOVA, followed by post hoc Tukey's test was used (SPSS, 16). Independent samples t test was used to designate significant differences in knee diameter, synovial level of IL-6, apoptotic markers, NF-кB, and FKN between groups. Significance level was set at $\mathrm{P} \leq 0.05$.

Results: The injection of Complete Freund's Adjuvant (CFA) caused intense knee edema $(\mathrm{P}<0.001)$, which was reduced by implementing anti-IL-6 $(\mathrm{P}<0.001)$, anti-FKN $(\mathrm{P}<0.001)$, Inh-NF-KB $(\mathrm{P}<0.001)$, and anti-FKN+ Inh NF-kB $(\mathrm{P}<0.001)$. The results indicated elevated levels of apoptotic markers during the acute phase $(\mathrm{P}=0.010)$, along with an increase in IL-6 $(\mathrm{P}<0.001)$, NF- $\mathrm{kB}$ $(\mathrm{P}<0.001)$, and FKN $(\mathrm{P}=0.030)$. Although IL-6 $(\mathrm{P}<0.001)$, NF-KB $(\mathrm{P}=0.001)$, and FKN $(\mathrm{P}=0.007)$ levels elevation continued during the chronic phase, the apoptosis markers decreased in this phase $(\mathrm{P}=0.050)$. The findings revealed that Anti-IL-6 treatment during different phases of the study could change the synovial NF-KB and FKN.

Conclusion: It seems that time-dependent variations in apoptotic markers level may be involved in pathogenesis of adjuvant-induced knee arthritis. In conclusion, synovial IL-6 through NF-кB- FKN pathway can play an important role in this process.

Keywords: Apoptosis, Adjuvant, Fractalkine, IL-6, Inflammation, NF-кB

Conflicts of Interest: None declared

Funding: This study was financially supported by the Neurophysiology Research Center, Shahid Beheshti University of Medical Sciences, Tehran, Iran.

\section{*This work has been published under CC BY-NC-SA 1.0 license. \\ Copyright $\subseteq$ Iran University of Medical Sciences}

Cite this article as: Golabi S, Zaringhalam J, Manaheji H. Synovial interlukin-6 affects apoptosis induction via nuclear factor kappa-B and fractalkine pathway during adjuvant arthritis. Med J Islam Repub Iran. 2020 (25 Mar);34:25. https://doi.org/10.47176/mjiri.34.25

\section{Introduction}

The process of programmed cell death, or apoptosis is recognized as a mechanism by which unwanted or useless cells are eliminated (1). Mitochondrial membrane disruption and leakage is the triggering step in apoptosis (2). Also, hypoxia can induce apoptosis (2), and antioxidant

Corresponding author: Dr Jalal Zaringhalam, jzaringhalam@sbmu.ac.ir

Physiology Department, School of Medicine, Shahid Beheshti University of Medical Sciences, Tehran, Iran

2. Abadan Faculty of Medical Sciences, Abadan, Iran

3. Neurophysiology Research Center, Shahid Beheshti University of Medical Sciences, Tehran, Iran enzymes inhibit apoptosis (2). Deregulation of apoptosis is the major pathophysiological mechanism of many autoimmune diseases (3). In addition, it was reported that apoptotic cells are much more prevalent in a variety of chronic inflammatory diseases such as rheumatoid arthri-

$\uparrow$ What is "already known" in this topic:

Cytokines and chemokines are major contributors to inflammation and apoptosis. Apoptosis disruptions play substantial roles in pathogenesis of arthritis and its symptoms.

\section{$\rightarrow$ What this article adds:}

Time-dependent variations in apoptotic markers level may be involved in pathogenesis of inflammation (Adjuvant-induced knee arthritis). Synovial IL-6 through NF-кB- FKN pathway can play an important role in this process. 
tis (RA), a chronic inflammatory disease characterized by synovial membrane hypertrophy with symptoms such as joint destruction, pain, and edema $(4,5)$. Furthermore, the hyperplasia of synovial cells in the joints of RA patients is a typical example of chronic inflammatory proliferation, which emphasizes the dysregulation of apoptosis in the pathophysiology of chronic inflammatory diseases (6). Cytokines are considered as important mediators of inflammatory situations. Some studies revealed that systemic and active inflammatory cytokines are produced abundantly within the patients' joints with $\operatorname{RA}(7,8)$. In addition, they are considered as important participants in the pathophysiology of chronic inflammatory diseases such as RA (9). IL-6 is a multifunctional, pro/anti-inflammatory cytokine, which plays some important roles in the pathophysiology of arthritis $(10,11)$. Some studies demonstrated that there is an extremely high IL-6 concentration within the serum and synovial fluid of RA patients (12). Furthermore, some reported that cytokines are involved in many cell processes such as differentiation, proliferation, and cell death (11). It is evident that cytokines can affect the extracellular pathway of apoptosis (1). IL-6, as an important inflammatory cytokine, has different antiapoptotic and proapoptotic roles. Some studies indicated that IL-6 can delay or prevent apoptotic cell death in some biological systems, while some suggested that IL-6 acts as an important tumor-promoting factor in a wide variety of human cancers, such as breast, glioma, and lymphoma cancers. These IL-6 effects are mediated through inhibiting apoptosis and promoting cell proliferation $(13,14)$. On the other hand, some researches emphasized the stimulatory effects of IL-6 on apoptosis. It was revealed that IL-6 stimulates neutrophil apoptosis during chronic inflammation. In this regard, IL- 6 can play an important role in the resolution of inflammation (15). In addition, different cell signaling pathways may be considered as an important part of various effects of cytokines during inflammation process. The results of some previous studies indicated anti/pro hyperalgesia effects of IL-6 during different stages of inflammation $(11,16)$. Cytokines exert some of their intracellular effects via chemokines (17). Like cytokines, chemokines are involved in the immune pathogenesis of inflammatory diseases, such as RA, and can affect apoptosis pathway $(7,18,19)$. FKN is described as the solitary member of a unique $\mathrm{CX} 3 \mathrm{C}$ class of chemokines (7), as well as a crucial mediator of apoptosis, which is upregulated in RA synovial fluid (SF) (7). In some studies, the effect of FKN on inducing or inhibiting apoptosis was confirmed, which is different due to kind and time of stimulation $(20,21)$. In addition, a part of intracellular effects of cytokines and chemokines are mediated via activating the NF-kB, which plays an important role in most immune and inflammatory responses, as well as the protection of cells from apoptosis $(22,23)$. An inappropriate regulation of $\mathrm{NF}-\mathrm{KB}$ activation has been involved in the pathogenesis of several immune related diseases such as RA (23). Some studies reported that chemokines are regarded as one of the NF-KB dependent gene products. For example, the stimulation of FKN by NF-KB dependent signals was shown during inflammatory situations (24,
25). Considering the significant effects of apoptosis on pathogenesis of inflammation and the key role of IL-6, NF-KB, and FKN pathway in inflammation and apoptosis, the present study was aimed to examine the role of synovial IL-6 in inducing apoptosis during different phases of adjuvant-induced arthritis and its relationship with synovial NF-кB- FKN signaling pathway.

\section{Methods}

\section{Animals and experimental groups}

Adult male Wistar rats, weighing 180-200 g, were used at the beginning of the experiments. The animals were housed at a RT of $22.0 \pm 1^{\circ} \mathrm{C}$, with a 12-h light-dark cycle. Food and water were available all the times except during the experiments. The study protocol was approved by the local ethics committee for using animals in research by following the guidelines of ethical standards for considering experimental pain in animals (26). Also, this study was approved by the ethics committee of Shahid Beheshti University of Medical Sciences (Thesis number: 13/1769). The experimental groups were arranged as follow: adjuvant induced arthritis (AIA), AIA control (mineral oil only), AIA + anti-IL-6, control + anti-IL-6 (PBS), AIA+ anti- fractalkine, control + anti-fractalkine (PBS), AIA + $\mathrm{NF}-\mathrm{kB}$ inhibitor, control+ NF-kB inhibitor (PBS), AIA + anti-fractalkine $+\mathrm{NF}-\mathrm{kB}$ inhibitor, and control + antifractalkine + NF-kB inhibitor (PBS). Every group was classified into 4 subdivisions to appraise diversities during the study period $\left(0,7^{\text {th }}, 14^{\text {th }}\right.$, and $21^{\text {st }}$ days $)$ and each subgroup included 6 male rats.

\section{Adjuvant-induced inflammation}

Adjuvant-induced inflammation was evoked on day 0 by a single intra-articular injection $(100 \mu \mathrm{L})$ of heat-killed Mycobacterium tuberculosis suspended in sterile mineral oil (10 mg/mL, CFA, Sigma, St Louis, MO, USA) into the right knee joint of the rat. AIA control rats received only sterile mineral oil $(100 \mu \mathrm{L})$ injection. This model was selected because it can exhibit a rapid primary inflammation response to the adjuvant (27).

\section{Evaluation of adjuvant-induced inflammation}

Adjuvant-induced inflammation development was evaluated by measuring the knee diameter during study period. Knee diameter changes on different days were indicated by using a Vernier caliper (28).

\section{Synovium extraction}

To study the molecular composition, the synovium of the knee joint in the rats was removed. To this end, the right kneecap of the rats was opened and the synovial tissue surrounding the enclosures was removed with precaution (29). Then, the synovium weight was calculated via scale instrumentation (A \& D Company, GR200, Japan). The obtained samples were first stored in a liquid nitrogen for 30 minutes and transferred to $-80^{\circ} \mathrm{C}$ freezer for later use.

\section{Synovial IL-6 measurement}

The synovial tissue was homogenized after segregation 
by the lysis buffer (Radio Immuno Precipitation Assay, RIPA, buffer). Synovial IL-6 level was evaluated by rat standard ELISA kit (Rat Interleukin-6 (IL-6). For the quantitative determination of rat IL6 in serum, plasma, tissue homogenates, cell lysates, cell culture supernates, and other biological fluids, Cat. No. KT-19418 was used on day 0 (before arthritis induction) and at different phases of the study according to the manufacturer's protocol. The collected solution showed $100 \%$ cross reactivity with the ELISA kit.

\section{Western blot analysis}

Western blot method was used to evaluate the synovial level of apoptotic markers (Bax, Bcl2, and Caspase3), as well as the synovial level of NF-kB and FKN. To this end, the rates were killed and the synovial tissue was quickly removed and homogenized in RIPA buffer. In the next stage, the synovial tissue was centrifuged, the supernatant was collected, and protein concentration was determined (30). The same quantity of protein was diluted with sample buffer and a fractional of the attenuated sample $(24 \mu \mathrm{L})$ was loaded onto SDS-PAGE gels and run. Sample from an individual synovium tissue was loaded on each lane. In the next stage, the proteins were transferred to ImmobilonP PVDF membrane (Millipore, Bed ford, MA) using miniPROTEAN II (Bio-Rad). After utilization of primary antibodies (Rabbit polyclonal IGg for Bax (1/ 1000), cell signaling; rabbit polyclonal IGg for Bcl2 (1/ 1000), cell signaling; rabbit polyclonal IGg for Caspase3 (1/ 1000), cell signaling; rabbit polyclonal IgG for NF-KB (1/3000), AbCam; goat polyclonal $\mathrm{IgG}$ for $\mathrm{CX} 3 \mathrm{CL} 1 /$ fractalkine (1/1000), R \& D systems), nonspecific binding sites on the membrane were blocked by the incubation in blocking buffer. Further, the membrane was washed 3 times with Tris Buffered Saline with Tween (TBST) buffer. Next, the membrane was incubated with secondary antibody (IgG $(1 / 3000)$, cell signaling for apoptotic indicators; antirabbit IgG (1/3000), cell signaling for nuclear factor kappa-B; and antigoat $\operatorname{IgG}(1 / 2000), \mathrm{AbCam} / \mathrm{CA}$ for Fractalkine). Then, the membrane was washed with TBST buffer 3 times. The immunoreactivity of the protein bands was observed using a chemiluminescence detection system (ECL, Amersham). Then, the membrane was incubated in a stripping buffer and with primary antibody for $\beta$-actin (Rabbit polyclonal IGg for $\beta$-actin (1/1000), cell signaling) as a loading control protein. In addition, the band congestion was quantified by NIH Image (1. 60) and presented as the ratio of the Bax, Bcl 2, Caspase 3, NF-KB and FKN bands to beta-actin to determine any discrepancies in starting Bax, Bcl 2, Caspase 3, Nuclear Factor kappa-B and fractalkine. Finally, each test was repeated 3 times on maiden groups of rats.

\section{Experimental manner}

Rats were randomly divided into different experimental groups (24 in each group). Each group was assigned into 4 subgroups with 6 male rats in each subgroup to evaluate variations on days $0,7,14$, and 21 .

Articular inflammation was developed by a single intraarticular injection of adjuvant in the right knee joint of the rat on day 0 (under light anesthesia) in experimental groups. In addition, different experiments were performed to determine the effects of serum IL-6, NF-KB, and FKN levels on knee diameter and find whether there was a time-dependent relationship. A neutralizing dose of AntiIL-6 (Anti-IL-6 Antibody, ab9770, Abcam/ CA, UK), Anti-FKN (CX3CL1/ fractalkine chemokine domain antibody R \& D, AF537) antibodies and Inh NF-kB (Cayman, USA, catalogue item No. 10012628) diluted in PBS were injected intraperitoneally $(11,31,32)$ from day 1 until the $21^{\text {st }}$ day of the study (weekly injection for Anti-IL-6 Antibody; 3 times a week injection for Anti-FKN antibody and Inh NF-KB inhibitor). As different phases were described for adjuvant-induced arthritis $(33,34)$, knee diameter, synovial IL-6, apoptotic markers, NF-kB, and FKN level evaluation were made on day 0 (immediately before CFA injection) and on days 7 (inflammatory phase), 14, and 21 (arthritic phase). Then, the rats were killed at the end of each experiment based on guidelines for Ethical Conduct in the Care and Use of Animals, and synovial membranes were parted, instantly frozen in liquid nitrogen, and stored at $-80^{\circ} \mathrm{C}$. In the next stage, synovial IL-6, apoptotic markers, NF-KB, and FKN levels were assessed by rat ELISA kit and Western blotting on different days of the study in the trial groups, respectively. The same manner was adopted for the control groups.

\section{Statistical analysis}

The study results were expressed as Mean \pm Standard Error of Mean (SEM). To analyze within-group variations, repeated measures ANOVA, followed by post hoc Tukey's through multiple comparison tests (SPSS, 16), were used. In addition, unpaired student $t$ test was applicate to designate considerable differences in knee diameter, synovial level of IL-6, apoptotic markers, NF-KB, and FKN between groups. Significance level was set at $p \leq 0.05$.

\section{Results}

\section{Knee size during study procedure}

Intra-articular adjuvant injection was shown to significantly increase the ipsilateral knee diameter until day 21 , compared to AIA control (mineral oil) group. In adjuvant injected rats, a significant increase occurred in the knee size during days $7(\mathrm{p}<0.001), 14(\mathrm{p}=0.001)$, and 21 $(p=0.001)$ after adjuvant injection, compared to day 0 . The results in the AIA+ anti-IL-6 group demonstrated that adjuvant-injected knee size could be significantly reduced by anti-IL-6 administration on days $7(\mathrm{p}<0.001)$ and 14 $(p=0.042)$, compared to the same days in the AIA group. Furthermore, anti-IL-6 administration caused a significant increase in knee diameter on day $21(\mathrm{p}=0.050)$, compared to the similar day in the AIA group.

With respect to the AIA+ anti-FKN group, anti-FKN antibody led to a significant decrease in knee size on days $7(\mathrm{p}<0.001), 14(\mathrm{p}=0.001)$, and $21(\mathrm{p}<0.001)$, compared to AIA group in the same days. Inh-NF- $\mathrm{KB}$ administration significantly reduced knee size on days $7(p<0.001), 14$ $(\mathrm{p}<0.001)$, and $21(\mathrm{p}<0.001)$, compared to the AIA group in the same days. However, no significant difference was 
observed between AIA and AIA+ PBS groups. Anti-FKN and Inh-NF-kB usage incurred a significant decline in knee size on days $7(p=0.001), 14(p=0.001)$, and 21 $(\mathrm{p}<0.001)$ of the study, compared to AIA group. However, no significant differences were reported between adjuvant injected rats and AIA+ PBS group (Table 1).
Synovial Bax/ Bcl2 changes during different phases of the study

Densitometry analysis revealed that intra-articular adjuvant injection could cause significant changes timedependently in synovial $\mathrm{Bax} / \mathrm{Bcl} 2$, compared to AIA control (mineral oil) group. The synovial $\mathrm{Bax} / \mathrm{Bcl} 2$ signifi-

Table 1. Knee diameter substantially increased in Adjuvant getter rats

\begin{tabular}{lccc}
\hline Groups & Day & Knee diameter mean $(\mathrm{mm})$ & SE \\
\hline CFA & 0 & 7.6 & 0.15 \\
& 7 & 12.4 & 0.53 \\
& 14 & 12.6 & 0.51 \\
CFA+ Anti IL-6 & 21 & 12.9 & 0.48 \\
& 0 & 7.7 & 0.17 \\
& 7 & 8.7 & 0.17 \\
CFA+ Anti FKN & 14 & 11.2 & 0.31 \\
& 21 & 14.4 & 0.44 \\
& 0 & 7.9 & 0.09 \\
CFA+ Inh NF-kB & 7 & 9.2 & 0.51 \\
& 14 & 9.5 & 0.52 \\
& 21 & 10.6 & 0.41 \\
CFA+ Anti FKN+ Inh NF-kB & 0 & 7.9 & 0.07 \\
& 7 & 9.2 & 0.49 \\
& 14 & 9.6 & 0.5 \\
& 21 & 9.8 & 0.51 \\
& 0 & 7.4 & 0.1 \\
\end{tabular}

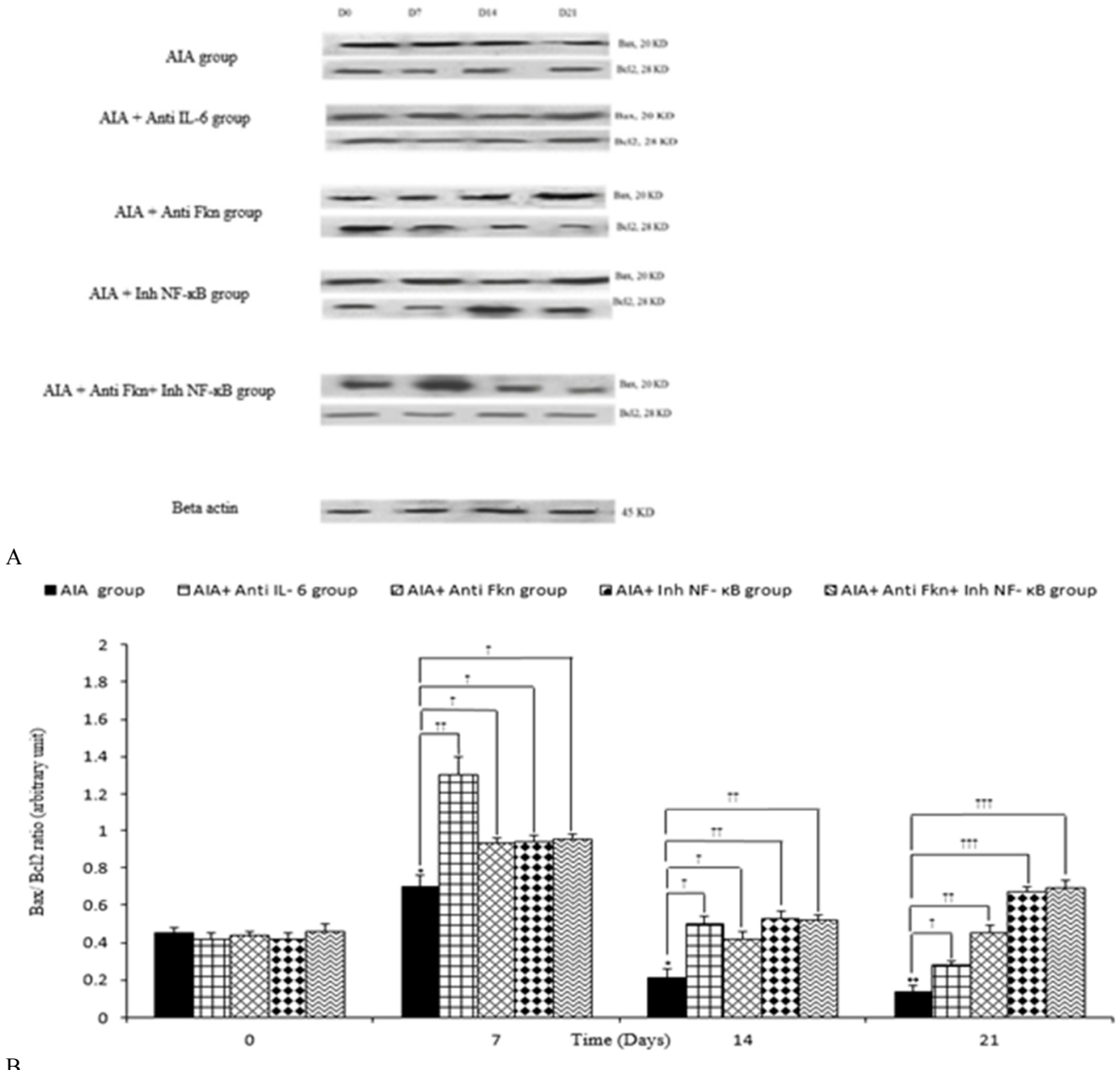

B

Fig. 1. Variation of synovial Bax/ Bcl 2 during different days of study

$4 \quad$ http://mjiri.iums.ac.ir

Med J Islam Repub Iran. 2020 (25 Mar); 34:25. 
cantly increased on day 7 in synovial membranes obtained from the adjuvant injected rats, compared to day 0 of the study $(\mathrm{p}=0.041)$. The synovial $\mathrm{Bax} / \mathrm{Bcl} 2$ during days 14 $(p=0.050)$ and $21(p=0.004)$ of adjuvant treatment was significantly lower than the amount during day 0 .

The administration of anti-IL-6 Antibody was associated with a prominent increment in synovial $\mathrm{Bax} / \mathrm{Bcl} 2$ on days $7(p=0.003), 14(p=0.050)$, and $21(p=0.047)$ of the study, compared to the similar days in AIA group. Treatment with anti-FKN antibody significantly increased the synovial $\mathrm{Bax} / \mathrm{Bcl} 2$ on days $7(\mathrm{p}=0.048), 14(\mathrm{p}=0.050)$, and $21(\mathrm{p}=0.005)$ of the study, compared to AIA group. Intraperitoneal utilization of NF-KB inhibitor led to a significant increase in synovial $\mathrm{Bax} / \mathrm{Bcl} 2$ on days 7 $(p=0.032), 14(p=0.010)$, and $21(p<0.001)$ of the study, compared to the similar days in AIA group. Administrating anti-FKN and Inh-NF-KB in adjuvant injected rats resulted in significantly increased synovial $\mathrm{Bax} / \mathrm{Bcl} 2$ on days $7(p=0.043), 14(p=0.050)$, and $21(p=0.006)$ of the study, compared to AIA group. However, no significant differences were observed between AIA and AIA+ PBS groups (Fig. 1a, b).
Synovial Caspase3/ 6-actin variation during study procedure

Intra-articular adjuvant injection could cause significant changes time-dependently in synovial caspase $3 / \beta$-actin, compared to the AIA control (mineral oil) group. A prominent enhancement occurred in caspase $3 / \beta$-actin on day 7 $(\mathrm{p}=0.010)$, while a significant decrease was observed on days $14(\mathrm{p}=0.050)$ and $21(\mathrm{p}=0.010)$, compared to day 0 .

Caspase $3 / \beta$-actin significantly increased in the synovial membrane of the AIA+ anti-IL- 6 group during days 7 $(p=0.001), 14(p=0.002)$, and $21(p=0.390)$, compared to the AIA group. Caspase $3 / \beta$-actin in the synovial membrane of the AIA+ Anti-FKN group significantly increased on days $7(\mathrm{p}=0.010), 14(\mathrm{p}=0.010)$, and 21 $(p=0.007)$ of the study, compared to the AIA group. Also, Inh-NF-KB injection significantly increased synovium level of Caspase 3/ $\beta$-actin during days $7(p=0.004), 14$ $(\mathrm{p}=0.050)$, and $21(\mathrm{p}=0.010)$ of the study, compared to AIA group. Caspase $3 / \beta$-actin in the synovial membrane of the AIA + anti-FKN+ Inh-NF-kB group significantly increased during days $7(\mathrm{p}=0.002), 14(\mathrm{p}=0.006)$, and 21 $(\mathrm{p}<0.001)$, compared to AIA group. There were no significant differences between AIA and AIA+ PBS groups (Fig. 2a, b).

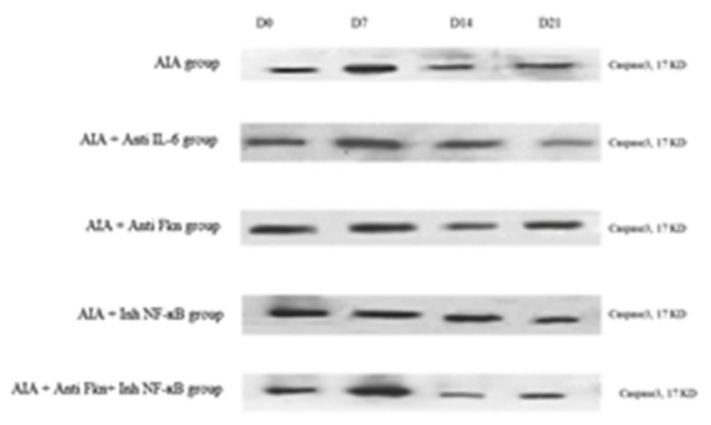

A
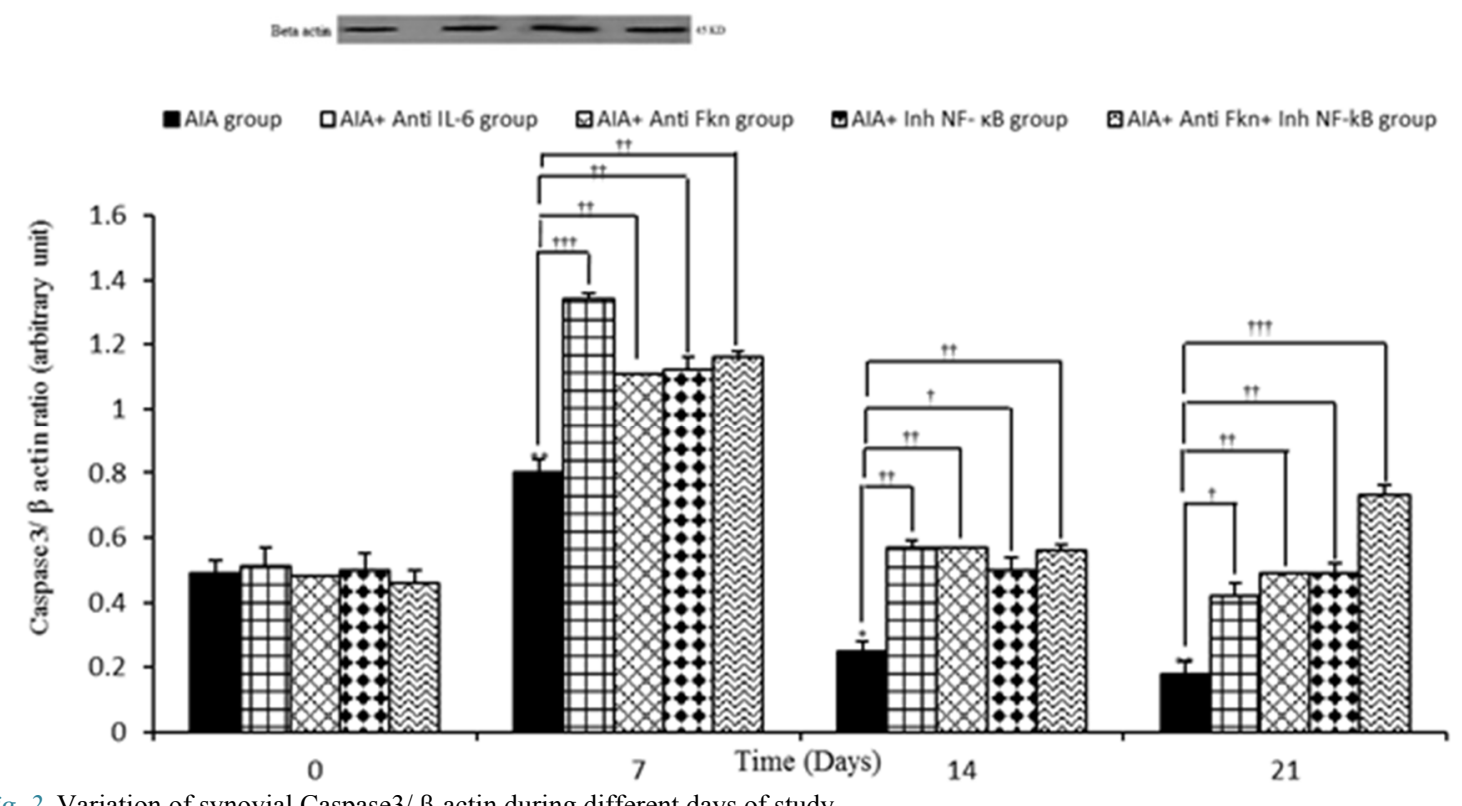

Fig. 2. Variation of synovial Caspase3/ $\beta$-actin during different days of study 
Synovial FKN/ 6-actin variation during study procedure

Intra-articular adjuvant injection was shown to cause considerable changes in synovial FKN/ $\beta$-actin, compared to the AIA control (mineral oil) group. The results also indicated that synovial FKN/ $\beta$-actin was significantly increased on days 7, 14, and 21 on the synovial membranes achieved from the adjuvant injected rats, compared to day 0 of the study ( $\mathrm{p}=0.020$ for days 7 and $14 ; \mathrm{p}=0.008$ for day 21). Treatment with Anti-FKN antibody significantly reduced synovial FKN/ $\beta$-actin on days 7 $(p=0.001), 14(p=0.008)$, and $21(p=0.010)$, compared to the AIA group. Administration of Anti-IL-6 antibody caused a reduction in synovial FKN/ $\beta$-actin in day 7 of the study and increased the ratio in days $14(\mathrm{p}=0.050)$ and $21(\mathrm{p}=0.010)$, compared to the AIA group.

Inh-NF-кB injection also significantly reduced synovial $\mathrm{FKN} / \beta$-actin on days $7(\mathrm{p}=0.050), 14(\mathrm{p}=0.003)$, and 21 $(\mathrm{p}=0.001)$, compared to AIA group on the same groups. Administration of Anti-FKN and Inh-NF-KB significantly decreased the synovial $\mathrm{FKN} / \beta$-actin during days 7 $(p=002), 14(p<0.001)$, and $21 \quad(p<0.001)$, compared to
AIA. However, there were no significant differences between AIA and AIA+ PBS groups (Fig. 3a, b).

Synovial NF-KB/ $\beta$-actin variation during study procedure

Intra-articular adjuvant injection led to considerable changes in synovium level of NF-KB/ $\beta$-actin, compared to AIA control (mineral oil) group.

Based on the synovial membranes obtained from adjuvant recipient rats, the synovium level of NF-KB/ $\beta$-actin was shown to considerably increase on days $7(\mathrm{p}=0.050)$, $14(\mathrm{P}=0.003)$, and $21(\mathrm{P}<0.001)$ of the study, compared to day 0 . Inh-NF-кB injection also significantly decreased the synovium level of NF-KB/ $\beta$-actin during days 7 $(\mathrm{p}=0.010), 14(\mathrm{p}=0.010)$, and $21(\mathrm{p}<0.001)$ of the study, compared to AIA group on the same days. Anti-IL-6 antibody administration was associated with decreased synovium level of NF-KB/ $\beta$-actin on days $7(p=0.049)$ and 14 $(\mathrm{p}=0.050)$, as well as in the ratio on the $21^{\text {st }}$ day $(\mathrm{p}=0.004)$, compared to the AIA group. There were no significant differences between AIA and AIA+ PBS groups (Fig. 4a, b).

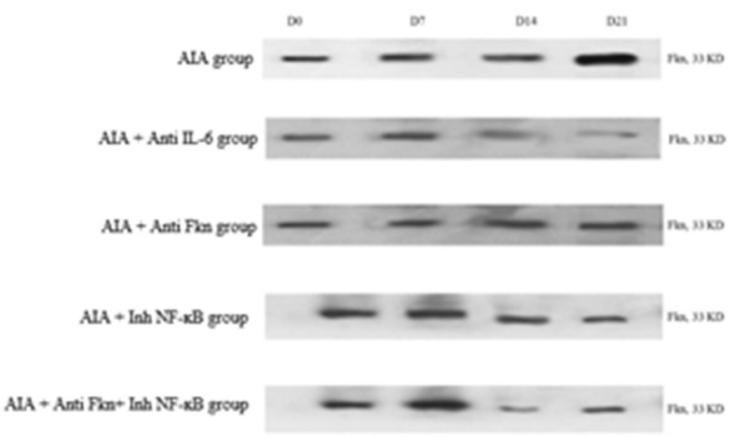

A

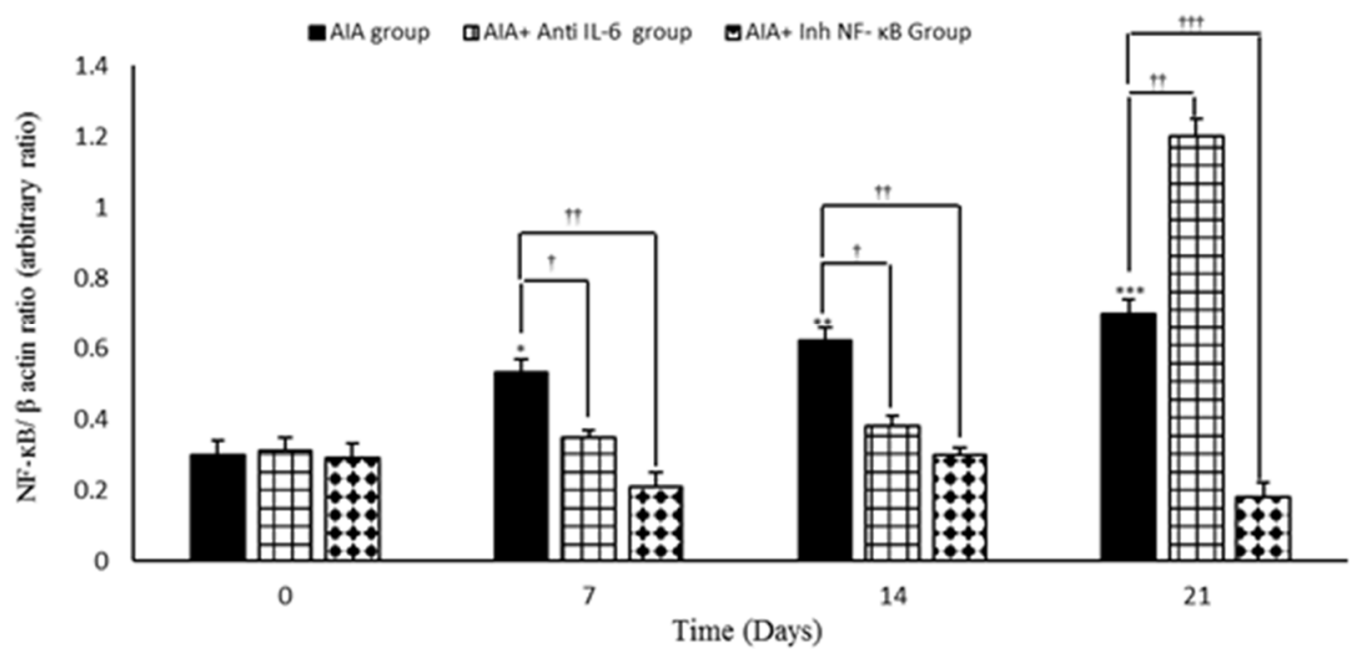

B

Fig. 3. Variation of synovial level of Fractalkine/ $\beta$-actin during different days of study 

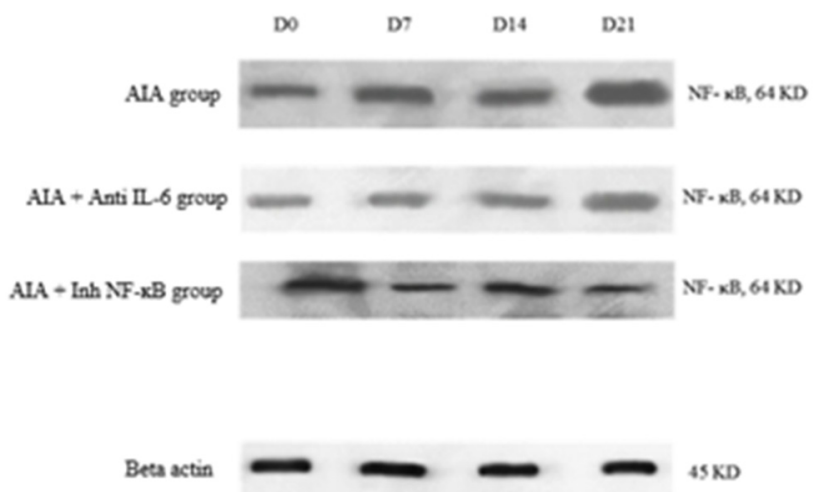

A

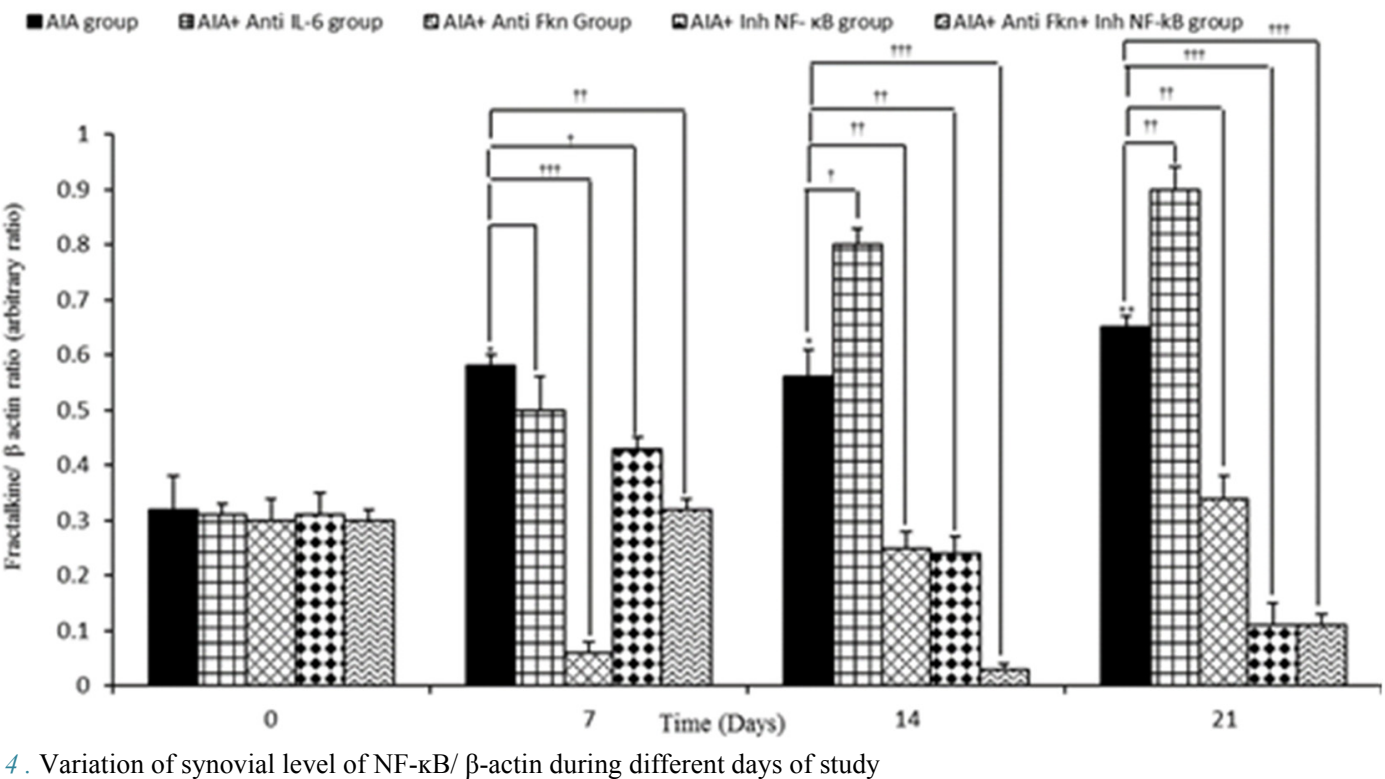

Fig. 4. Variation of synovial level of NF-кB/ $\beta$-actin during different days of study

Synovial IL-6 level variation during study procedure

A significant increase was observed in IL-6 concentration after adjuvant treatment, compared to the AIA control (mineral oil) group. In addition, the synovial IL-6 level significantly increased on days $7(\mathrm{p}<0.001), 14(\mathrm{p}=0.001)$, and $21(\mathrm{p}<0.001)$, compared to day 0 in the adjuvant injected rats. Administration of anti-IL-6 antibody returned

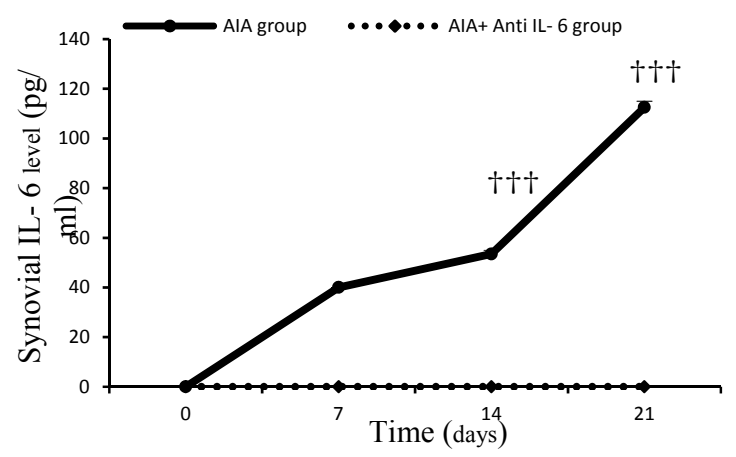

Fig. 5. ELISA analysis of synovium level of IL-6 during different phases of study synovial IL-6 levels to those of day 0 before adjuvant injection, and the AIA+ anti-IL-6 rats indicated a significant reduction in synovial IL-6 level, compared to the similar days in the AIA group ( $\mathrm{p}<0.001$ for all). No significant difference was observed between AIA and AIA+ PBS group (Fig. 5).

No differences were found between AIA, AIA control, and AIA+ PBS groups in different parts of the study. Only the AIA group results are presented in figures.

\section{Discussion}

The results indicated that intra-articular adjuvant injection led to permanent synovial edema. In addition, some changes were observed in synovial apoptotic markers during different stages of adjuvant-induced knee arthritis, and IL-6 played an important role in the above-mentioned changes via NF-kB- FKN signaling pathway. Further, the findings demonstrated that synovial FKN plays a substantial role in inducing and progressing knee edema and varying synovial apoptotic markers created by IL-6 and NF-KB during adjuvant-induced arthritis. Some studies demonstrated that positional adjuvant approval is one of the prevalent scientific methods for evaluating inflammatory 
disturbances, such as RA, and can be used to effectively assess various behavioral and molecular changes in acute and chronic inflammatory arthritis $(34,35)$. Furthermore, it was reported that cytokines and chemokines play a significant role in pathogenesis of inflammation, as well as its related symptoms such as hyperalgesia and edema (1, $35)$. In addition, arthritis results in increasing the levels of inflammatory mediators such as IL- 6 , TNF- $\alpha$ and IL-1 $\beta$ $(36,37)$. Based on the results of this study, injecting intraarticular adjuvant can increase synovial IL-6 level align through increasing knee edema, which is in line with the results of some other studies which demonstrated that adjuvant plantar injection elevates serum IL-6 level during the whole 21 days (11). Further, the findings demonstrated that administering anti-IL-6 antibody weekly can decrease knee edema during the first 2 weeks, while an increase occurs in knee edema during the chronic phase of arthritis due to anti-IL-6 antibody treatment. The results can suggest dual anti/ proinflammatory roles for synovial IL-6 during adjuvant-induced arthritis (11).

Furthermore, adjuvant-induced arthritis caused an elevation in synovial apoptotic indicators during the acute stage of the study (Day 7). However, these indicators were shown to decrease on days 14 and 21 , compared to day 0 . These results were in accordance with the results of studies showing that apoptosis level in RA synovium relies considerably on the disease stage (38). Furthermore, in another study, alveolar epithelial cells became apoptotic and inflammatory mediators could promote the induction of apoptosis during acute lung inflammation (39). Moreover, apoptosis elevated during acute kidney inflammation due acute ischemic or nephrotoxic damage (40). In addition, chronic inflammatory bowel diseases can predispose an individual to cancer by inducing cell resistance to apoptosis, which is similar to the results of the present study (41). Further, inflammatory disorders can change apoptosis pattern, which results in influencing inflammatory contexts. It was revealed that apoptosis defects can lead to the induction of autoimmune disorders such as RA. Furthermore, the activation of apoptotic pathways may play a considerable role in improving inflammatory symptoms $(42,43)$. Inflammatory cytokines play a substantial role in modulating apoptosis. By the same token, the apoptotic markers could increase during the first week of inflammation in the present study, which is in line with an increase in the synovial IL-6 level. In this regard, some studies highlighted the significance of IL-6 in inflammatory late phases. For example, Ferraccioli et al demonstrated that the early phases of the disease seemed to be characterized by a systematic increase in IL- $1 \beta$ and TNF- $\alpha$ in AIA model of arthritis, while the late phases were characterized by an increase in IL-6, so that IL-6 effects on apoptosis and inflammatory symptoms can be more manifested during chronic inflammation (44).

On the other hand, the results of the present study indicated a decrease in apoptotic markers during the last 2 weeks, along with an increase in synovial IL-6 level. In addition, administering weekly anti-IL-6 antibody could decrease synovial IL- 6 level, leading to a significant increase in synovial apoptotic markers. Further, some re- search emphasized IL-6 stimulatory effect on apoptosis. It was reported that the human promonocytic leukemic cell line and mature human neutrophils can be induced to become apoptotic when cultured with interleukin-6. Proapoptotic effect of IL-6 was evident after 48 hours of cell incubation with IL-6 (45). Since the importance of IL6 in the transition from acute to chronic phase of inflammation was emphasized, its effects on apoptosis are more considerable in the chronic phase than in acute the arthritis phase $(46,47)$. In addition, some emphasized the antiapoptotic role of IL- 6 by indicating that IL- 6 can act as a growth factor for epidermal keratinocyte cells, mesenchymal cells, renal carcinoma cells, and multiple myeloma cells $(48,49)$. Further, IL-6 can inhibit apoptosis induction during inflammation via 2 distinct pathways, including NF-кB-dependent (NF-kB/ FKN) and NF-кB- independent pathway. Through these 2 pathways, IL- 6 can prevent from inducing apoptosis $(12,50)$. Some reports on NF-KB, as one of the most important intracellular signaling pathways in IL-6 $(17,51)$, which are congruent with the results of the present study, indicated that adjuvant-induced inflammation increased synovial NF-KB level during the whole study period. Anti-IL-6 antibody treatment resulted in reducing synovial IL-6 and NF-KB level significantly. Furthermore, NF-KB could cause cellular signaling through varying FKN expression. In addition, an increased activation of NF-KB can increase the expression level of FKN $(17,51)$, which is consistent with the results of the present study, which indicated an increase in synovial FKN, along with an increase in the synovial level of NFкB. Furthermore, NF-кB inhibitor administration caused a considerable decrease in synovial FKN level and a significant reduction in synovial NF-KB during the whole study. In an in vitro study, it was reported that the FKN expression induced in rat aortic endothelial cells correlated with an increase in NF-KB activity, and NF-кB inhibition resulted in preventing from inducing FKN expression (12). Furthermore, based on the results of the present study, reducing the level of synovial FKN due to NF-KB inhibition was consistent with increasing apoptotic markers. The findings confirmed that direct inhibition of administering FKN by Anti-FKN antibody and its indirect inhibition by NF-KB inhibitor administration caused a significant increase in synovial apoptotic markers. The results of this study can be used for drawing the pathway of inhibitory effects in IL-6 on apoptosis induction during chronic inflammation. IL-6 neutralizing can lead to a reduction in synovial NF-KB and FKN levels and the induction of synovial apoptosis.

\section{Conclusion}

Based on the results, an increased level of synovial IL$6, \mathrm{NF}-\mathrm{KB}$, and FKN can play a significant role in inducing inflammation and changing the apoptosis pattern during adjuvant-induced arthritis. The findings confirmed that IL6 has time-dependent effects on synovial apoptosis induction and NF-KB- FKN pathway is regarded as a crucial part of its anti-apoptotic effects during chronic inflammation. Thus, given the importance of apoptosis deregulation during chronic inflammation, the evaluation of other 
pathways is recommended for further studies.

\section{Acknowledgments}

The present study was supported by Neurophysiology Research Center of Shahid Beheshti University of Medical Sciences.

\section{Financial and ethical issues}

This study was financially supported by the Neurophysiology Research Center of Shahid Beheshti University of Medical Sciences.

\section{Conflict of Interests}

The authors declare that they have no competing interests.

\section{References}

1. Lockshin RA, Zakeri Z. Apoptosis, autophagy, and more. Int J Biochem Cell Biol. 2004;36:2405-2419.

2. Amani H, Habibey R, Hajmiresmail SJ, Latifi Sh, Pazoki-Toroudi H, Akhavan O. Antioxidant nanomaterials in advanced diagnoses and treatments of ischemia reperfusion injuries. J Mater Chem B. 2017;5:9452-9476.

3. Hayashi T, Faustman D. Role of defective apoptosis in type 1 diabetes and other autoimmune diseases. Recent Prog Horm Res. 2003;58:131-153.

4. Foyet HS, Tsala DE, Bodo JCZE, Carine AN, Fabrice NH, Heroyne LT, et al. Anti-arthritic and anti-inflammatory propensity of catechins from Vitellaria paradoxa. Pharmacogn Res. 2014;7:367-377.

5. William-Vandivier R, Henson PM, Douglas IS. The impact of failed apoptotic cell removal (Efferocytosis) on chronic inflammatory lung disease. Chest. 2006;129:1673-1682.

6. Yoshida K, Ochiai A, Matsuno H, Gabriel S, Panayi GS, Corrigall VM. Binding immunoglobulin protein resolves rheumatoid synovitis: a xenogeneic study using rheumatoid arthritis synovial membrane transplants in SCID mice. Arthritis Res Ther. 2011;13:1-6.

7. Ruth JH, Volin MV, Haines GK, Woodruff DC, Katschke KJ, Woods JM, et al. Fractalkine, a novel chemokine in rheumatoid arthritis and in rat adjuvant-induced arthritis. Arthritis Rheum. 2001;44:15681581.

8. Vervoordeldonk MJBM, Tak PP. Cytokines in rheumatoid arthritis. Curr Rheumatol Rep. 2002;4:208.

9. Feldmann M, Maini SRN. Role of cytokines in rheumatoid arthritis: an education in pathophysiology and therapeutics. Immunol Rev. 2008;223:7-19.

10. Hwang SY, Kim JY, Kim KW, Park MK, Moon Y, Kim WU, et al. IL-17 induces production of IL-6 and IL-8 in rheumatoid arthritis synovial fibroblasts via NF-KB and PI3-kinase/ Akt-dependent pathways. Arthritis Res Ther. 2004;6:R120-R128.

11. Tekieh E, Zaringhalam J, Manaheji H, Maghsoudi N, Alani B, Zardooz H. Increased serum IL-6 level time-dependently regulates hyperalgesia and spinal mu opioid receptor expression during CFAinduced arthritis. EXCLI J. 2011;10:23-33.

12. Rose-John S, Scheller J, Elson G, Jones SA. Interleukin-6 biology is coordinated by membrane-bound and soluble receptors: role in inflammation and cancer. J Leukocyte Biol. 2006;80:227-236.

13. Takeda K, Kaisho T, Yoshida N, Takeda J, Kishimoto T, Akira SH. Stat3 activation is responsible for IL-6-dependent $T$ cell proliferation through preventing apoptosis: generation and characterization of $\mathrm{T}$ cell-specific Stat3-deficient mice. J Immunol. 1998;161:4652-4660.

14. Waldner MJ, Foersch S, Neurath MF. Interleukin-6- a key regulator of colorectal cancer development. Int J Biol Sci. 2012;8:1248-1253.

15. Kaplanski G, Marin V, Montero-Julian F, Mantovani A, Farnarier C. IL-6: a regulator of the transition from neutrophil to monocyte recruitment during inflammation. Trends Immunol. 2003;24:25-29.

16. Tekieh E, Zaringhalam J, Akhtari Z. Relationship between cytokines and spinal mu opioid receptor expression during adjuvant-induced arthritis in rats. Annu Res Rev Boil. 2014;4:1854-1866.

17. Garcia GE, Xia Y, Chen S, Wang Y, Ye RD, Harrison JK, et al. NFkappa B-dependent Fractalkine induction in rat aortic endothelial cells stimulated by IL-1beta, TNF-alpha, and LPS. J Leukoc Biol. 2000;67:577-84

18. Kastenbauer S, Koedel U, Wick M, Kieseier BC, Hartung HP, Pfister HW. CSF and serum levels of soluble Fractalkine (CX3CL1) in inflammatory diseases of the nervous system. J Neuroimmunol. 2003; $137: 210-217$

19. Mizuno T, Kawanokuchi J, Numata K, Suzumura A. Production and neuro protective functions of Fractalkine in the central nervous system. Brain Res. 2003;979:65-70.

20. Lin MT, Juan CHY, Chang KJ, Chen WJ, Kuo ML. IL-6 inhibits apoptosis and retains oxidative DNA lesions in human gastric cancer AGS cells through up-regulation of anti-apoptotic gene mcl-1. Carcinogenesis. 2001;22:1947-1953.

21. Isozaki $T$, Kasama $T$, Takahashi R. Synergistic induction of CX3CL1 by TNF alpha and IFN gamma in osteoblasts from rheumatoid arthritis: involvement of NF-kappa B and STAT-1 signaling pathways. J Inflamm Res. 2008;1:19-28.

22. Makarov SS. NF-kB as a therapeutic target in chronic inflammation: recent advances. Mol Med Today. 2000;6:441-448.

23. Roman-Blas JA, Jimenez SA. NF-KB as a potential therapeutic target in osteoarthritis and rheumatoid arthritis. Osteoarthr Cartil. 2006;14:839-848.

24. Lawrence T. The nuclear factor NF-kB pathway in Inflammation. Cold Spring Harb Perspect Biol. 2009;1:1-10.

25. Guo X, Pan Y, Xiao C, Wu Y, Cia D, Gu J. Fractalkine stimulates cell growth and increases its expression via NF-KB pathway in RAFLS. Int J Rheum Dis. 2012;15:322-329.

26. Zimmermann M. Ethical guidelines for investigations of experimental pain in conscious animals. Pain. 1983;16:109-110.

27. Santora K, Rasa C, Visco D, Steinetz BG, Bagnell CA. Antiarthritic effects of relaxin, in combination with estrogen, in rat adjuvantinduced arthritis. J Pharmacol Exp Ther. 2007;322:887-893.

28. Multag SH. Dose dependent anti-inflammatory effect of Ammi majus alcoholic extract in rat: chronic study. Iraqi $\mathrm{J}$ Pharm Sci. 2012;21:82-86.

29. Hyc A, Osiecka-Iwan A, Dziunycz P, Moskalewski S. Preparation of rat synovial membrane for studies of cytokine secretion. Folia Histochem Cytobiol. 2007;45:57-60.

30. Bradford MM. Rapid and sensitive method for the quantitation of microgram quantities of protein utilizing the principle of protein- dye binding. Anal Biochem. 1976;72:248-254.

31. Suzuki F, Nanki T, Imai T, Kikuchi H, Hirohata Sh, Hitoshi Kohsaka H, Miyasaka N. Inhibition of CX3CL1 (Fractalkine) Improves Experimental Autoimmune Myositis in SJL/J Mice. J Immunol. 2005;175(10):6987-6996.

32. González-Ramos R, Van Langendonckt A, Defrère S, Lousse JC, Mettlen M, Guillet A, Donnez J. Agents blocking the nuclear factorkappaB pathway are effective inhibitors of endometriosis in an in vivo experimental model. Gynecol Obstet Invest. 2008;65(3):174-86.

33. Cicala C, Innaro A, Fiorucci S. No-naproxen modulates inflammation, nociception and downregulates $\mathrm{T}$ cell response in rat Freund's adjuvant Arthritis. Br J Pharmacol. 2000;130:490-7.

34. Taniguchi N, Kanai S, Kawamoto M, Endo H, Higashino H. Study on application of static magnetic field for adjuvant arthritis rats. J Evid Based Complementary Altern Med. 2004;1:187-191.

35. Zaringhalam J, Tekieh E, Manaheji H, Akhtari Z. Cellular events during arthritis-induced hyperalgesia is mediated by Interleukin- 6 and p38 MAPK and their effects on the expression of spinal mu-opioid receptors. Rheumatol Int. 2013;33:2291-2299.

36. Rodriguez Vita J, Lawrence T. The resolution of inflammation and cancer. Cytokine Growth Factor Rev. 2010;21:61-65.

37. Jones B, Koch AE, Ahmed S. Pathological role of Fractalkine/CX3CL1 in rheumatic diseases: a unique chemokine with multiple functions. Front Immunol. 2012;2:1-22.

38. Catrina AI, Ulfgren AK, Lindblad S, Grondal L, Klareskog L. Low levels of apoptosis and high FLIP expression in early rheumatoid arthritis synovium. Ann Rheum Dis. 2002;61:934-936.

39. Matute-Bello G, Winn RK, Jonas M, Chi EY, Martin TR, Conrad Liles W. Fas (CD95) induce alveolar epithelial cell apoptosis in vivo. Am J Pathol. 2001;158:153-161.

40. Lieberthal W. Biology of acute renal failure: therapeutic implications. Kidney Int. 1997;52:1102-1115.

41. Shacter E, Weitzman SA. Chronic inflammation and cancer. Oncology. 2002 16:217-26.

42. Ramos MV, Fernández GC, Brando RJ, Panek CA, Bentancor LV, 
Landoni VI, et al. Interleukin-10 and interferon- $\gamma$ modulate surface expression of Fractalkine-receptor (CX3CR1) via PI3K in monocytes. Immunology. 2010;129:600-609.

43. Zhang N, Yin Y, Xu SHJ, Wu YP, Chen WSH. Inflammation \& apoptosis in spinal cord injury. Indian J Med Res. 2012;135:287-296.

44. Ferraccioli G, Bracci-Laudiero L, Alivernini S, Gremese E, Tolusso B, De Benedetti F. Interleukin- $1 \beta$ and Interleukin-6 in arthritis animal models: roles in the early phase of transition from acute to chronic inflammation and relevance for human rheumatoid arthritis. Mol Med. 2010;16:552-557.

45. Afford SC, Pongracz J, Stockley RA, Crocker J, Burnett D. The induction by human interleukin- 6 of apoptosis in the promonocytic cell line U937 and human neutrophils. J Biol Chem. 1992;267:2161221616.

46. Torpy DJ, Papanicolaou DA, Lotsikas AJ, Wilder RL, Chrousos GP, Pillemer SR. Responses of the sympathetic nervous system and the hypothalamic-pituitary-adrenal axis to interleukin-6. Arthritis Rheum. 2000;43:872-80.

47. Nishimoto N. Kishimoto T. Interleukin 6: from bench to bedside. Nat Rev Rheumatol. 2006;2:619-26.

48. Puthier D, Bataille R, Amiot M. IL-6 up-regulates Mcl-1 in human myeloma cells through JAK/STAT rather than Ras/MAP kinase pathway. Eur J Immunol. 1999;29:3945-3950.

49. Xu G, Zhang Y, Zhang L, Ren G, Shi Y. The role of IL-6 in inhibition of lymphocyte apoptosis by mesenchymal stem cells. Biochem Biophys Res. 2007;361:745-750.

50. Libermann T, Baltimore D. Activation of interleukin- 6 gene expression through the NF-kappa B transcription factor. Mol Cell Biol. 1990;10:2327-34.

51. Hohmann HP, Brockhaus M, Baeuerle PA, Remy R, Kolbeck R, Van Loon AP. Expression of the types A and B Tumor Necrosis Factor (TNF) receptors is independently regulated, and both receptors mediate activation of the transcription factor NF-kappa B. TNF alpha is not needed for induction of a biological effect via TNF receptors. J Biol Chem. 1990;265:22409-22417. 